\section{Hanf-Zigaretten als Verkaufsschlager}

Sie riechen und schmecken wie ein Joint, sollen aber nicht high machen: Hanf-Zigaretten sind in der Schweiz ein Verkaufsschlager. In Deutschland ist das Produkt allerdings tabu, warnt der Zoll. Das Schweizer Bundesamt für Gesundheit ist besorgt, dass die Hanf-Zigarette jungen Leuten als Einstiegsdroge dienen könnte. „Weil HanfZigaretten den gleichen Geschmack haben wie Drogenhanf, kann ein gewisser Gewöhnungseffekt nicht ausgeschlossen werden“, sagte Pressesprecher Daniel Dauwalder. „Da Hanf-Zigaretten immer auch ein wenig THC enthalten, ist nicht ganz auszuschließen, dass z.B. Neukonsumenten eine leicht berauschende Wirkung verspüren.“

www.aerztezeitung.de

\title{
Kommission fordert Verbot neuer Droge Drogen!
}

Die Europäische Kommission will die Designerdroge Furanyl-Fentanyl verbieten lassen. Die neuartige psychoaktive Substanz Furanyl-Fentanyl ist laut Brüsseler Behörde „ein hoch potentes synthetisches Opioid, das dem Bericht der Europäischen Beobachtungsstelle für Drogen und Drogensucht (EBDD) zufolge zu erheblichen Schäden für die menschliche Gesundheit führen kann“. In Deutschland seien bisher mit dem Konsum von Furanyl-Fentanyl vier Todesfälle in Verbindung gebracht worden, EU-weit gingen 23 Tote auf das Konto der Designerdroge. Furanyl-Fentanyl werde typischerweise als Pulver oder gebrauchsfertiges Nasenspray verkauft.

Seit März 2017 dürfen Ärzte zu medizinischen Zwecken Cannabisblüten und -extrakt verschreiben und bestimmten Patienten so auf legalem Weg das Leben erleichtern. Weitaus häufiger ist jedoch der illegale und gefährliche Konsum des Rauschgifts. Lesen Sie im Schwerpunkt "Suchtmedizin" mehr zum Thema Substanzmissbrauch! In dieser Ausgabe können Sie auBerdem kräftig CMEPunkte sammeln: Mit jeweils 6 und 4 Punkten ist die Weiterbildung garantiert!

Claudia Daniels Redakteurin

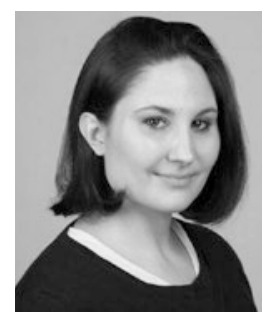

\section{Voll Speed zum frühen Schlaganfall}

Alkoholsucht trifft immer häufiger auch Frauen. „War ein problematisches Trinkverhalten zu Beginn des letzten Jahrhunderts bei Männern noch dreimal so verbreitet wie bei Frauen, lagen Männer zum Ende des 20. Jahrhunderts nur noch geringfügig vorne", erklärt Dr. Bastian Willenborg, Berlin. Insbesondere auf junge Menschen treffe das zu: Bei zwischen 1991 und 2000 Geborenen bestünden kaum noch geschlechtsspezifische Unterschiede. Die Beobachtungen Willenborgs werden von $\mathrm{Da}$ ten aus dem jüngst vorgestellten Drogenund Suchtbericht gestützt. Während die

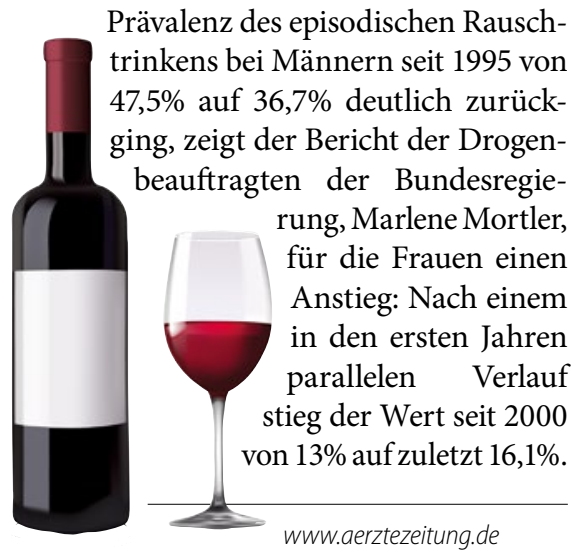

\section{Zahl der computersüchtigen Jugendlichen steigt an}

Jugendliche müssen frühzeitig - etwa im schulischen Kontext - im bewussten Umgang mit Medien geschult werden, um dem signifikanten Anstieg bei Computerspiel- und Internetsucht entgegenzuwirken. Daran erinnerte die Bundeszentrale für gesundheitliche Aufklärung (BZgA) zum Auftakt der Computerspielmesse "gamescom“ in Köln. Aktuelle Zahlen der BZgA unterstreichen das Problem auch im jüngsten Drogen- und Suchtbericht von Marlene Mortler (CSU), Drogenbeauf- tragte der Bundesregierung. Im Jahr 2015 ist nach den Befunden der BZgA-Drogenaffinitätsstudie demnach bei 5,8\% aller 12 bis 17-Jährigen von einer Computerspieloder Internetabhängigkeit auszugehen. Unter jungen Erwachsenen zwischen 18 und 25 Jahren sind es mit 2,8\% deutlich weniger Betroffene. Laut Mortler müssten auch Eltern „wachsam bleiben und ein medienkompetentes Vorbild sein“.

www.bzga.de
Junge Menschen, die Methamphetamin konsumieren, laufen Gefahr, „vom Schlag getroffen zu werden“. Überwiegend handelt es sich um hämorrhagische Insulte. Vollständige Erholung ist die Ausnahme. Eine Arbeitsgruppe hat für eine Übersichtsarbeit zu diesem Thema 77 Artikel ausgewertet, in denen über Schlaganfälle von 98 Personen unter 45 Jahren im Zusammenhang mit dem Konsum von Amphetamin oder Methamphetamin berichtet wurde. 81 der Insulte waren hämorrhagischer Natur. Der Anteil hämorrhagischer Schlaganfälle in Verbindung mit Methamphetamin bzw. Amphetamin ist mit $80 \%$ ungewöhnlich hoch. Zwar ist der Anteil zerebraler Hämorrhagien an allen Insulten bei jüngeren Menschen generell höher. Allerdings wird die Rate gewöhnlich auf 40-55\% taxiert. Über alle Altersgruppen hinweg liegt der Anteil bei 15-20\%. Die Forscher blicken mit einiger Sorge in die Zukunft; angesichts des steigenden Amphetaminkonsums würden damit verbundene Schlaganfälle zunehmen. Außerdem verweisen sie auf die schlechte Prognose von Insulten unter Amphetamin.

Lappin JM et al. J Neurol Neurosurg Psychiatry 2017 (online first) 